\title{
ATTENTION DEFICIT HYPERACTIVITY DISORDER
}

\section{DEVELOPMENTAL COORDINATION DISORDER AND ADHD}

The prevalence, comorbidity, and outcome of "developmental coordination disorder (DCD)" were studied in 7-10 year-old children at the Department of Child and Adolescent Psychiatry, Sahlgren University Hospital, Goteborg, Sweden. Motor examinations by teacher and physician comprised 11 tests that included, hopping, alternating movements, finger tapping, and finger imitation. Behavior was observed and scored according to attention deficit, hyperactivity, impulsivity, and distractibility. Moderate to severe symptoms of ADHD were noted in 50\%.

Twenty children ( $5 \%$ of the population) - 18 boys, 2 girls - had severe DCD, and 35 (9\%) - 29 boys, 6 girls - had moderate DCD; a DCD rate of 8-13\% among boys and $1-3 \%$ in girls, and a male-female ratio of $4-7: 1$. Signs of DCD were stable during the follow-up period from 7 through 10 years of age. School dysfunction score, ranging from 0 to 10 , was 1 in the non-DCD group, 4 in the moderate, and 5 in the severe DCD group. DCD with ADHD children had a mean score of 6 . Both DCD and ODD were strongly comorbid with ADHD. DCD and ADHD, alone or combined, and showed an increased correlation with Asperger's disorder. DCD carried an increased risk of speech-language and reading problems, and DCD with comorbid ADHD had the lowest reading scores. (Kadesjo B, Gillberg C. Developmental coordination disorder in Swedish 7-year-old children. L Am Acad Child Adolesc Psychiatry July 1999;38:820-828). (Reprints: Dr Gillberg, Department of Child and Adolescent Psychiatry, Sahlgren University Hospital, S-413 45 Goteborg, Sweden).

COMMENT. The diagnostic criteria for developmental coordination disorder, listed in the 1987 DSM-III-R, include 1) marked impairments of performance in daily activities requiring motor coordination, having regard to age and IQ (delayed motor milestones, clumsiness, dysgraphia, and poor sports abilities); 2) impaired academic achievement or activities of daily living; and 3) not due to cerebral palsy or muscular dystrophy. The term "developmental" is, in fact, a misnomer, since the etiology in some cases may relate to birth anoxia, ischemia, and prematurity, or to postnatal factors. In the pre-DSM-III Psychiatric Association terminology, DCD would have been known as the "clumsy child syndrome," "motor-perception disorder," and "minimal brain dysfunction," now termed ADHD.

A careful neurologic examination of children presenting with ADHD will frequently uncover dyspraxias, synkinesias, motor impersistence, utilization behaviors, dysdiadochokinesia, choreiform movements, graphanesthesia, and other minimal abnormalities consistent with DCD. It is not surprising that children diagnosed as DCD would show comorbidity with ODD and problems in behavior, attention, and school achievement. In the next revised issue of the American Psychiatric Association DSM, perhaps the neurological signs characterized as DCD could be included among the criteria for the diagnosis of $\mathrm{ADHD}$, at least as an additional sub-type. Neurologists should be more involved with the ADHD diagnostic criteria and their management.

\section{SPEECH AND LANGUAGE DEVELOPMENT IN ADHD}

Speech discrimination and phonological working memory were examined in children with $\mathrm{ADHD}(\mathrm{N}=9)$, ADHD plus developmental coordination disorder $(\mathrm{ADHD}+\mathrm{DCD})(\mathrm{N}=13)$, and 19 age-matched controls, in a study at the Neuropediatric Unit, Karolinska Institute, Stockholm, Sweden. Tests requiring monosyllabic discrimination showed no significant differences between subject groups, 
whereas the ADHD+DCD group scored significantly lower in tests involving exposure to multisyllabic (2-5) non-word pairs and speech discrimination with a working-memory load. The low performance in the ADHD children with complicating DCD was the result of impaired memory rather than of speechdiscrimination problems. (Norrelgen F, Lacerda F, Forssberg H. Speech discrimination and phonological working memory in children with ADHD. Dev Med Child Neurol May 1999;41:335-339). (Respond: Dr F. Norrelgen, MotorikLab, Astrid Lindgrens Children's Hospital, Stockholm, S-171 76, Sweden).

COMMENT. Language problems are reported in $65 \%$ of children with ADHD and motor-perception impairments (DCD), and hyperactive behavioral disorders affect approximately the same proportion of children with learning disabilities. Phonological awareness and working memory are important in the acquisition of reading and writing skills. The present study suggests that deficits in working memory may contribute to the high incidence of dyslexia among children with $\mathrm{ADHD}$, and may explain their motor-perception impairments by effects on storage of motor commands at an early age. See Denckla MB, In Progress in Pediatric Neurology II, 1994;pp173-176, for an account of the phonological-linguistic basis of dyslexia, its anatomic-phsiologic correlates, and the overlap between reading disability and other learning disabilities with "ADD $(\mathrm{H}) . "$

\section{METHYLPHENIDATE IN ADHD PRESCHOOLERS WITH DISABILITY}

The safety and efficacy of methylphenidate (MPH), 0.3 and $0.6 \mathrm{mg} / \mathrm{kg}$, in 11 preschool children (aged 4-5 years) with developmental disabilities and attention deficit hyperactivity disorder (ADHD) were determined in a double-blind, placebocontrolled, crossover study at the Western Psychiatric Institute, University of Pittsburgh, PA. IQs ranged from 40 to 78 (mean, 60). No child had autism/PDD. A positive response to MPH was obtained in $73 \%$. Teacher ratings of hyperactivity and inattention and clinic-based observations of compliance and activity level were significantly improved. Adverse MPH side effects in 5 (45\%) patients included severe social withdrawal, crying, and irritability. (Handen BL, Feldman HM, Lurier A, Murray PJH. Efficacy of methylphenidate among preschool children with developmental disabilities and ADHD. LAm Acad Child Adolesc Psychiatry July 1999;38:805-812). (Reprints: Dr Handen, Merck Program, Western Psychiatric Institute and Clinic, 3811 O'Hara St, Pittsburgh, PA 15213).

COMMENT. Preschool children with ADHD and developmental disabilities respond to $\mathrm{MPH}$ at rates similar to those of school-age children with mental retardation and $\mathrm{ADHD}$, but the risk of adverse drug effects is greater. Social withdrawal and irritability are frequent, especially at higher doses. A combination approach, behavioral intervention and lower MPH dose, might reduce the risk of side effects.

Are stimulants overprescribed? is addressed in a study of the diagnosis and treatment of ADHD in four US urban/suburban communities, including Puerto Rico (Jensen PS et al. I Am Acad Child Adolesc Psychiatry July 1999;38:797-804). Only $12.5 \%$ of children with a diagnosis of ADHD had received stimulant medication in the previous 12 months. Mental health counseling and/or schoolbased interventions were used more often than medication. Stimulants are not overprescribed, and their potential effectiveness may be neglected due to cultural or physician bias or lack of parent education. 